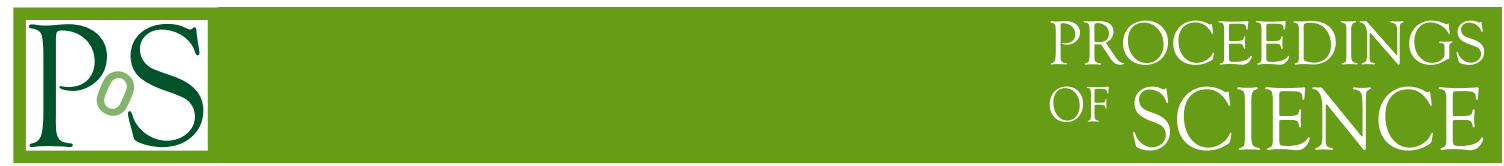

\title{
Top models of diffractive scattering to falsify at the LHC
}

\section{Anton Godizov*}

Institute for High Energy Physics, 142281 Protvino, Russian Federation

E-mail: anton.godizov@gmail.com

The talk is a review of current situation with theoretical description of the soft elastic scattering at the LHC and, in general, adresses the question why do we need diffractive studies at high energy hadron colliders.

LHC on the March,

November 16-18, 2011

Protvino, Moscow region, Russian Federation

\footnotetext{
* Speaker.
} 

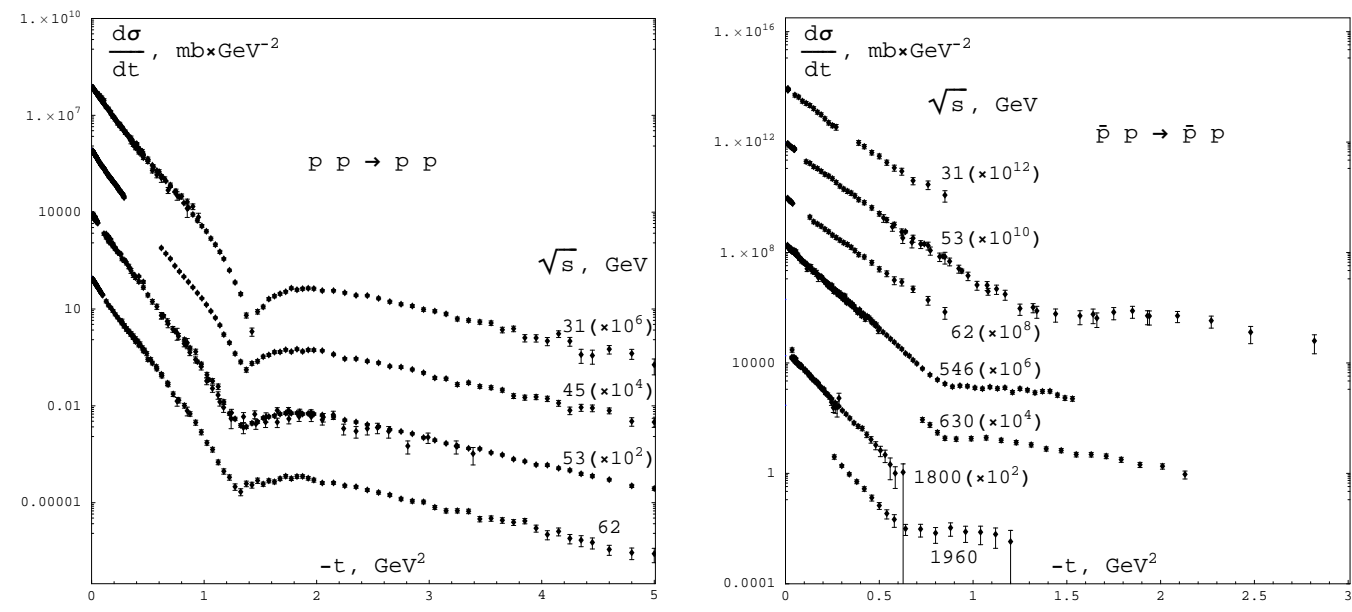

Figure 1: The diffractive pattern observed at high energy hadron colliders.

\section{Introduction}

Usually high energy physics is considered as the synonym of "physics of particles". New phenomena in this area are related to discoveries of new (mostly heavy) particles or to the typical "particle-like" effects, such as "Bjorken scaling" in Deep Inelastic Scattering or jets with high transverse momenta and so on. In the space-time language these kinematical regimes correspond to interaction at small distances.

However, there is an area in high energy physics, which is related to rather large distances, even at ultra-high energies. These phenomena concern the scattering of hadrons at small angles. The well-known feature of such processes is that the corresponding angular distributions [1] often reveal the typical diffractive behavior with zero-angle maximum (like those ones in Fig. 1). These reactions are called "diffractive".

Such a behavior of angular distributions points to the fact that experiments devoted to the study of diffractive processes are characterized first of all by the dominance of the wave aspects of the quantum-mechanical particle-wave duality. This fact determines their special status in the whole LHC physics program. In many ways this field is reminiscent of optics where the wave scattering is used to define the form, the size and the structure of the scatterer from the characteristic diffractive patterns.

The longitudinal and the transverse sizes of the hadron interaction region can be estimated through the Heisenberg uncertainty relations

$$
R_{L} \sim \Delta x_{L} \geq \frac{\sqrt{s}}{\sqrt{\left.<t^{2}>-<t\right\rangle^{2}}}, \quad R_{T} \sim \Delta x_{T} \geq \frac{1}{\sqrt{-<t>}}
$$

and the extraction of these quantities from the experimental elastic angular distributions can be done without theoretical assumptions (here $\sqrt{s}$ is the collision energy of hadrons, $\sqrt{-t}$ is the transverse momentum transfer and brackets denote averaging over the angular distribution). For example, at Tevatron the corresponding longitudinal size is larger than $5000 \mathrm{fm}$ and the transverse size is about $1 \mathrm{fm}$. Such a huge values point to the fact that diffractive phenomena are related rather to 

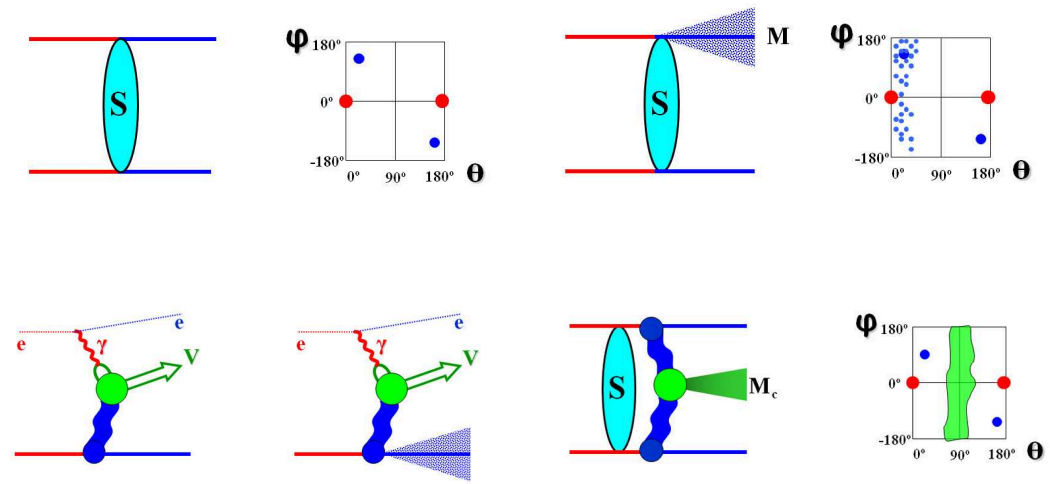

Figure 2: The typical diffractive processes.

wave behavior of hadrons than to their partonic structure. In other words, diffractive studies are not destined for the study of the details of the inner hadron structure.

It is evident now that (contrary to a rather widespread opinion) high energies do not always make corresponding cross-sections perturbatively calculable. To get an access to small distances the high collision energy is not enough, one needs comparably high momentum transfer. Otherwise (and this is exactly the case of diffractive processes), the smallness of the QCD "running" coupling does not help. Technically, this means that we are in the so-called "non-perturbative regime" related to quite large space-time intervals.

Two additional arguments in favor of diffractive studies are:

- the interaction at large distances is (in any case) related to the problem of confinement of quarks and gluons,

- at high-energy scattering the fraction of diffractive events in the total number of events is so significant that understanding of physical mechanisms of diffractive interaction is indispensable for general understanding of the strong interaction.

Hence, the importance of diffractive studies at high energy colliders is out of question. This field is one of the greatest challenges to both the theoretical and the experimental high energy physics communities.

\section{Theoretical framework for description of diffractive scattering}

The typical diffractive reactions are (Fig. 2):

- elastic scattering (when both participating particles remain intact) - for studying the size and the shape of hadrons at high energies;

- single diffraction (with the dissociation of one of the colliding particles) - for example, for studying the physics of the proton vacuum excitations;

- diffractive electroproduction of vector mesons on protons (this example illustrates the fact that hadron diffraction plays important role not only in hadron-hadron collisions); 
- exclusive central production of heavy mass states - for studying the interplay of short and large distance regimes.

All these processes are the sources of valuable information on the structure and properties of the hadron interaction region and QCD dynamics at large distances.

But in what way is it possible to connect diffractive pattern with the theory? QCD, at its modern stage, has no essential progress outside the perturbative calculations. It does not give any definite prediction, say, for the energy dependence of the total or differential cross-sections of proton-proton scattering. Nonetheless, one can trace some important contacts of experiment and theory.

This contact is accomplished with the help of Regge trajectories - analytic continuations of the resonance spectra [2]. Below in formulae (2.1)—(2.3) there is a recipe for calculation of elastic scattering amplitude in the framework of the Regge-eikonal approach.

$$
\begin{gathered}
T_{12 \rightarrow 12}(s, t)=4 \pi s \int_{0}^{\infty} d b^{2} J_{0}(b \sqrt{-t}) \frac{e^{2 i \delta_{12 \rightarrow 12}(s, b)}-1}{2 i}, \\
\delta_{12 \rightarrow 12}(s, b)=\frac{1}{16 \pi s} \int_{0}^{\infty} d(-t) J_{0}(b \sqrt{-t}) \delta_{12 \rightarrow 12}(s, t), \\
\delta_{12 \rightarrow 12}(s, t)=\sum_{n} \xi^{+}\left(\alpha_{n}^{+}(t)\right) \beta_{n}^{+}(t) s^{\alpha_{n}^{+}(t)} \mp \sum_{n} \xi^{-}\left(\alpha_{n}^{-}(t)\right) \beta_{n}^{-}(t) s^{\alpha_{n}^{-}(t)} .
\end{gathered}
$$

Here $T$ is the elastic scattering amplitude, the eikonal $\delta$ is the sum of single-reggeon-exchange terms, $s$ and $t$ are the Mandelstam variables, $b$ is the impact parameter, $\xi$ are signature factors, $\alpha(t)$ are famous Regge trajectories and $\Gamma(t)$ are reggeon form-factors of colliding particles.

At ultra-high energies only few terms in the eikonal survive and, so, the practical use of this approach is that in the case of high energy diffraction it allows to reduce the unknown function of two variables, $T_{12 \rightarrow 12}(s, t)$, to few functions of one dynamical variable $t$ (Regge trajectories and reggeon form-factors of the colliding particles) and to make explicit estimations for the high energy evolution of the diffractive pattern.

Regge trajectories (and, also, reggeon form-factors) should be determined from the relevant quantum field theory which is quantum chromodynamics. But in the framework of perturbative QCD one can calculate Regge trajectories at very high momentum transfers only [3]. This region of momentum transfer, however, gives a negligible contribution to total and elastic cross-sections.

Hence, one is enforced to invent "plausible" models which bear at least general QCD properties as much as possible.

\section{Models of high energy hadron diffraction vs. TOTEM}

The vast majority of phenomenological models [4], which pretend to the description of elastic diffraction, use the above-mentioned Regge-eikonal approach (or its modifications) as the theoretical framework. Some of these models are called "QCD-inspired", some of them do not appeal to QCD at all but all of them give predictions to differential and total cross-sections at ultra-high energies. The recent results of measurements implemented by the TOTEM Collaboration [5] allow experimental verification of these theoretical predictions (Tab. 1, Fig. 3). The TOTEM data on 


\begin{tabular}{|c|c|}
\hline The Model & $\sigma_{t o t}^{p p}(7 \mathrm{TeV}), \mathrm{mb}$ \\
\hline A. Donnachie, P.V. Landshoff, Phys.Lett. B 296 (1992) 227 & 91 \\
\hline P. Desgrolard, M. Giffon, L.L. Jenkovszky, Z.Phys. C 55 (1992) 637 & $87.5(6 \mathrm{TeV})$ \\
\hline P. Desgrolard, M. Giffon, E. Martynov, Eur. Phys. J. C 18 (2000) 359 & 95 \\
\hline V.A. Petrov, A.V. Prokudin, Eur. Phys. J. C 23 (2002) 135 & $97 \pm 4$ \\
\hline C. Bourrely, J. Soffer, T.T. Wu, Eur. Phys. J. C 28 (2003) 97 & 93 \\
\hline $\begin{array}{l}\text { R.F. Avila, S.D. Campos, M.J. Menon, J. Montanha, } \\
\text { Eur. Phys. J. C } 47 \text { (2006) } 171\end{array}$ & 94 \\
\hline M.M. Islam, R.J. Luddy, A.V. Prokudin, Int. J. Mod. Phys. A 21 (2006) & 97.5 \\
\hline E. Martynov, Phys. Rev. D 76 (2007) 074030 & 91 \\
\hline R.F. Avila, P. Gauron, B. Nicolescu, Eur. Phys. J. C 49 (2007) 581 & 108 \\
\hline E. Martynov, B. Nicolescu, Eur. Phys. J. C 56 (2008) 57 & 95 \\
\hline C. Flensburg, G. Gustafson, L. Lönnblad, Eur. Phys. J. C 60 (2009) 233 & $98 \pm 9$ \\
\hline P. Brogueira, J. Dias de Deus, J. Phys. J 37 (2010) 075006 & 110 \\
\hline M.M. Block, F. Halzen, Phys. Rev. D 83 (2011) 077901 & $95.5 \pm 1$ \\
\hline $\begin{array}{l}\text { L.L. Jenkovszky, A.I. Lengyel, D.I. Lontkovskyi, } \\
\text { Int. J. Mod. Phys. A } 26 \text { (2011) } 4755\end{array}$ & $98 \pm 1$ \\
\hline E. Gotsman, E. Levin, U. Maor, Eur. Phys. J. C 71 (2011) 1553 & 91 \\
\hline M.G. Ryskin, A.D. Martin, V.A. Khoze, Eur. Phys. J. C 71 (2011) 1617 & 89 \\
\hline S. Ostapchenko, Phys. Rev. D 83 (2011) 014018 & 93 \\
\hline $\begin{array}{l}\text { D.A. Fagundes, E.G.S. Luna, M.J. Menon, A.A. Natale, } \\
\text { arXiv: } 1108.1206 \text { [hep-ph] }\end{array}$ & 97 \\
\hline A. Godizov, Phys. Lett. B 703 (2011) 331 & 110 \\
\hline The TOTEM Collaboration, Europhys.Lett. 96 (2011) 21002 & $98.3 \pm 0.2^{\text {stat }} \pm 2.8^{\text {syst }}$ \\
\hline
\end{tabular}

Table 1: Comparison of the model predictions for the total $p p$ cross-section at $\sqrt{s}=7 \mathrm{TeV}$ with the TOTEM results.

the $p p$ cross-sections (especially on the differential one) reveal a very strong discriminative power. Contrary to that, the predictive power of all the considered models turnes out very weak. It means the fact that many of these models give a fine description of differential cross-sections from ISR to Tevatron energies (with the collision energy increased by several orders of magnitude). And though the ratio of the LHC energy to Tevatron energy is less than 4, we can observe a huge discrepancy between model curves and the data (for some models - by tens percent, for others - by several times).

But what are the consequences of such a result for QCD? The main surprise is that we came to the situation when the falsification of all the models did not influence the theory of strong interaction at all. We are still at the same stage of our misunderstanding of hadron diffraction as 30-40 years ago. Such a situation is quite a unique in high energy physics.

Of course, the job made by the TOTEM experimentalists is beyond any praise. And the significance of the TOTEM measurements is great since it disclosed an extremely unsatisfatory state 

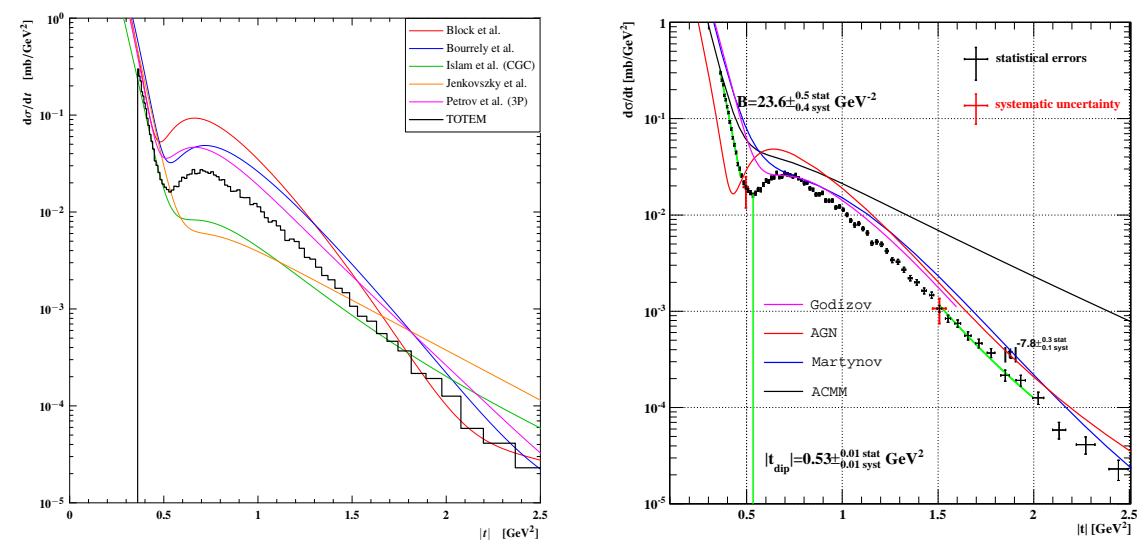

Figure 3: Comparison of the model predictions for the differential $p p$ cross-section at $\sqrt{s}=7 \mathrm{TeV}$ with the TOTEM results.

of the theory of diffractive interactions.

However, for not to end up with such a pessimistic note that everything is bad in the theory of diffractive interaction it would be appropriate to mention two experimental scenarios relevant for diffractive physics which could give some important information largerly independent of current theoretical insinuations.

\section{Diffraction at CMS}

Since quite a long time the question of universality of strong interaction was being discussed. Some authors considered that at high energies cross-sections will retain the memory of the quark content of colliding hadrons so that, say, at asymptotically high energies the ratio $\sigma_{\text {tot }}^{\pi N} / \sigma_{\text {tot }}^{N N}$ will tend to $2 / 3$ while others argued that all ratios would tend to the unity. In order to resolve the dispute one has to have very high-energy pion beams. At present time this is technically unattainable. Nonetheless, there are some ways to extract [6] (Fig. 4), say, $\pi N$ and even $\pi \pi$ cross-sections from the spectra of leading neutrons which are to be recorded at the Zero Degree Calorimeter (ZDC). In principle, this can give access to pion virtual Şbeams $\breve{T}$ in the $\mathrm{TeV}$ region of energies. It is very important, since for now we have experimental data on these cross-sections only up to $40 \mathrm{GeV}$ of the collision energy.

The second proposal for the CMS made by several authors [7] concerns the opportunity to arrange an interplay of typically diffractive process of the small-angle proton scattering with a typically "hard" process of the Higgs boson production in the pomeron-pomeron collisions (Fig. 5). In the course of the high-energy quasi-elastic scattering of the LHC protons they invest some part of their energies into the vacuum fluctuation giving rise, in particular, to the elusive Higgs boson. The main merit of such a diffractive manner to hunt for the Higgs boson is its cleanness because the heavy particle is to be produced not in the environment of other particles but in a comfortable loneliness, separated from two final protons by two significant rapidity gaps.

The corresponding measurements could be implemented with using such CMS detectors as the Forward Shower Counters (FSC, for detecting rapidity gaps in diffractive events and establishing 


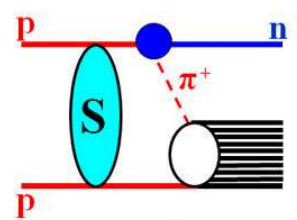

a)

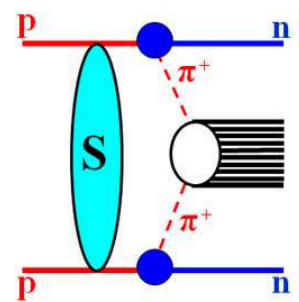

d)

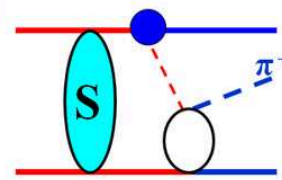

b)

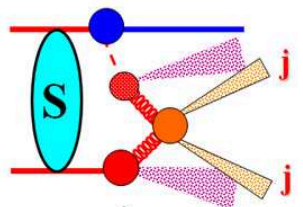

c)

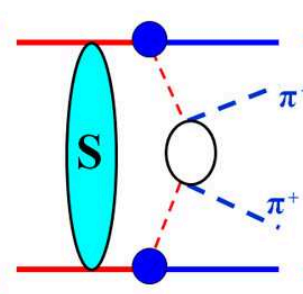

e)

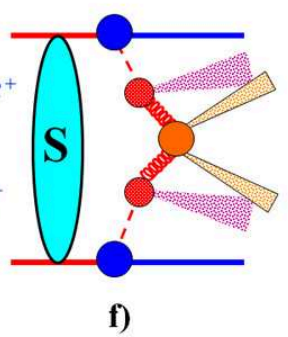

Figure 4: The extraction of the $\pi N$ and $\pi \pi$ cross-sections from the spectra of leading neutrons.

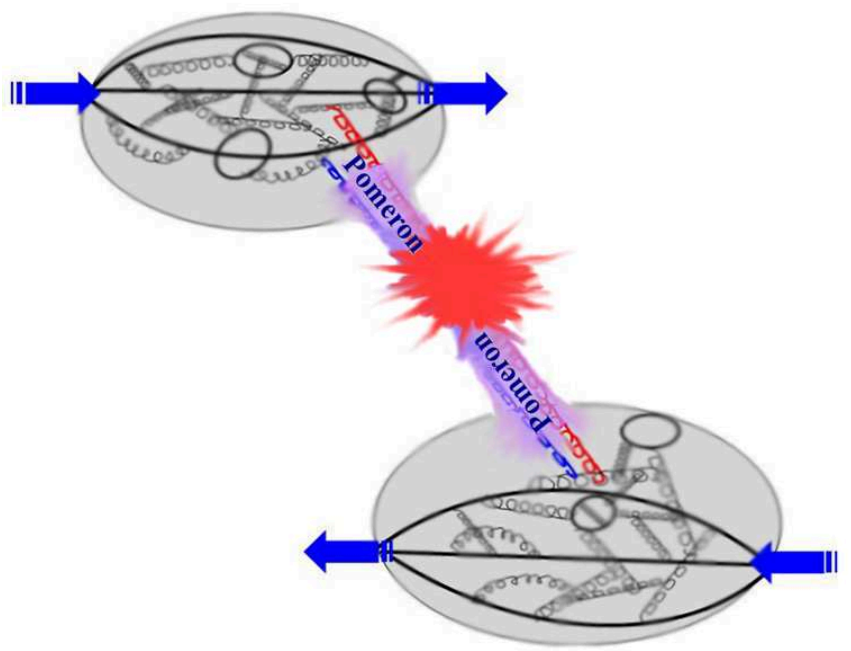

Figure 5: The exclusive diffractive central production of heavy mass state.

exclusivity in central exclusive channels) and the High Precision Spectrometer (HPS, for measuring the final proton momenta).

\section{Conclusion}

To conclude one has to acknowledge that the domain of diffractive studies is one of the most interesting and powerful parts of the whole LHC physics program. However, the progress in our understanding of diffractive interactions at high energies will be possible only after construction of more adequate phenomenological schemes than the existing ones. 


\section{References}

[1] Durham Hepdata Database, http://hepdata.cedar.ac.uk/; U. Amaldi and K. R. Schubert, Nucl. Phys. B 166, 301 (1980); A. Breakstone et al., Nucl. Phys. B 248, 253 (1984); N. Amos et al., Nucl. Phys. B 262, 689 (1985); UA1 Collab. (G. Arnison et al.), Phys. Lett. B 128, 336 (1983); UA4 Collab. (M. Bozzo et al.), Phys. Lett. B 147, 385 (1984); UA4 Collab. (M. Bozzo et al.), Phys. Lett. B 155, 197 (1985); UA4 Collab. (D. Bernard et al.), Phys. Lett. B 198, 583 (1987); UA4 Collab. (D. Bernard et al.), Phys. Lett. B 171, 142 (1986); E-710 Collab. (N. Amos et al.), Phys. Lett. B 247, 127 (1990); CDF Collab. (F. Abe et al.), Phys. Rev. D 50, 5518 (1994); D0 Collaboration, D0 Note 6056-CONF.

[2] P. D. B. Collins, //An Introduction to Regge Theory \& High Energy Physics. Cambridge University Press, 1977.

[3] J. Kwiecinski, Phys. Rev. D 26, 3293 (1982); R. Kirschner and L. N. Lipatov, Z. Phys. C 45, 477 (1990).

[4] A. Donnachie and P. V. Landshoff, Nucl. Phys. B 267, 690 (1986); L L. Jenkovszky,

A. M. Shelkovenko, and B.V. Struminsky, Z. Phys. C 36, 495 (1987); P. Desgrolard, M. Giffon, and L. L. Jenkovszky, Z. Phys. C 55, 637 (1992); P. Desgrolard, M. Giffon, and E. Martynov, Eur. Phys. J. C 18, 359 (2000); V. A. Petrov and A. V. Prokudin, Eur. Phys. J. C 23, 135 (2002); C. Bourrely, J. Soffer, and T. T. Wu, Eur. Phys. J. C 28, 97 (2003); R. F. Avila, S. D. Campos, M. J. Menon, and J. Montanha, Eur. Phys. J. C 47, 171 (2006); M. M. Islam, R. J. Luddy, and A. V. Prokudin, Int. J. Mod. Phys. A 21, 1 (2006); E. Martynov, Phys. Rev. D 76, 074030 (2007); R. F. Avila, P. Gauron, and B. Nicolescu, Eur. Phys. J. C 49, 581 (2007); E. Martynov and B. Nicolescu, Eur. Phys. J. C 56, 57 (2008); C. Flensburg, G. Gustafson, and L. Lönnblad, Eur. Phys. J. C 60, 233 (2009); P. Brogueira and J. Dias de Deus, J. Phys. J 37, 075006 (2010); M. M. Block and F. Halzen, Phys. Rev. D 83, 077901 (2011); L. L. Jenkovszky, A. I. Lengyel, and D. I. Lontkovskyi, Int. J. Mod. Phys. A 26, 4755 (2011); E. Gotsman, E. Levin, and U. Maor, Eur. Phys. J. C 71, 1553 (2011); M. G. Ryskin, A. D. Martin, and V. A. Khoze, Eur. Phys. J. C 71, 1617 (2011); S. Ostapchenko, Phys. Rev. D 83, 014018 (2011); D. A. Fagundes, E. G. S. Luna, M. J. Menon, and A. A. Natale, Testing parameters in an eikonalized dynamical gluon mass model, arXiv: 1108.1206 [hep-ph]; A. Godizov, Phys. Lett. B 703, 331 (2011).

[5] The TOTEM Collaboration, Europhys. Lett. 95, 41001 (2011); The TOTEM Collaboration, Europhys. Lett. 96, 21002 (2011);

[6] V. A. Petrov, R. A. Ryutin, and A. E. Sobol, Eur. Phys. J. C 65, 637 (2010); A. E. Sobol, V. A. Petrov, R. A. Ryutin, and M. J. Murray, Eur. Phys. J. C 69, 641 (2010).

[7] V. A. Khoze, A. D. Martin, and M. G. Ryskin, Eur. Phys. J. C 14, 525 (2000); V. A. Petrov and R. A. Ryutin, JHEP 0408, 013 (2004); V. A. Petrov and R. A. Ryutin, J. Phys. G 35, 065004 (2008). 